\title{
HIV Infection and TLR Signalling in the Liver
}

\author{
Megan Crane, ${ }^{1,2}$ Kumar Visvanathan, ${ }^{3}$ and Sharon R. Lewin ${ }^{1,2,4}$ \\ ${ }^{1}$ Department of Medicine, Monash University, Melbourne, VIC, Australia \\ ${ }^{2}$ Centre for Virology, Burnet Institute, Melbourne, VIC, Australia \\ ${ }^{3}$ Department of Medicine, Monash Medical Centre, Melbourne, VIC, Australia \\ ${ }^{4}$ Infectious Disease Unit, The Alfred, Melbourne, VIC, Australia
}

Correspondence should be addressed to Sharon R. Lewin, sharon.lewin@monash.edu

Received 26 August 2011; Accepted 29 October 2011

Academic Editor: Fabio Marra

Copyright () 2012 Megan Crane et al. This is an open access article distributed under the Creative Commons Attribution License, which permits unrestricted use, distribution, and reproduction in any medium, provided the original work is properly cited.

Despite the availability of effective combination antiretroviral therapy (cART), liver disease is one of the leading causes of morbidity and mortality in Human Immunodeficiency Virus (HIV)-infected individuals, specifically, in the presence of viral hepatitis coinfection. HIV, a single stranded RNA virus, can bind to and activate both Toll-like receptor (TLR)7 and TLR8 in circulating blood mononuclear cells, but little is known about the effect of HIV on TLRs expressed in the liver. HIV can directly infect cells of the liver and HIV-mediated depletion of CD4+ T-cells in the gastrointestinal tract (GI tract) results in increased circulating lipopolysaccharide (LPS), both of which may impact on TLR signaling in the liver and subsequent liver disease progression. The potential direct and indirect effects of HIV on TLR signaling in the liver will be explored in this paper.

\section{Introduction}

There are 33 million people infected with human immunodeficiency virus (HIV) and despite the availability of effective combination antiretroviral therapy (cART), life expectancy in HIV-infected patients remains reduced. Liver-related mortality is now the commonest cause of non-AIDS related death in HIV-infected individuals on cART $[1,2]$. Hepatitis B virus (HBV) or hepatitis $\mathrm{C}$ virus (HCV) are the main causes of liver disease in HIV-infected patients [3-6] with more rapid liver disease progression and higher liver-related mortality compared with individuals infected with either HBV or HCV alone [3-6].

Recently, there have been increasing reports of liver disease in HIV-infected individuals in the absence of viral hepatitis [7-11]. These diseases largely include liver decompensation with and without evidence of cirrhosis, nonalcoholic liver disease (NALD) its more severe form nonalcoholic steatohepatitis (NASH) and hepatocellular cancer (HCC) [711]. Liver disease in HIV monoinfection has been significantly associated with high HIV RNA, prolonged exposure to cART as well as high body mass index (BMI), alcohol abuse and increasing age [12-14].
This paper will discuss the likely direct and indirect effects of HIV on the innate immune system of the liver and the potential contribution of these changes to liver disease in the presence and absence of coinfection with hepatitis $\mathrm{B}$ and C.

\section{Toll-Like Receptor (TLR) Signalling and the Liver}

Toll-like receptors (TLRs) are pattern recognition receptors that recognize pathogen-associated molecular patterns (PAMPS). All TLRs have a cytosolic Toll/IL-1 receptor (TIR) domain, which is responsible for signal transduction. Myeloid differentiation primary response gene 88 (MyD88) is the main TIR-domain containing adapter molecules common to all TLRs except TLR3. Other TIR-domain containing adapter molecules include Toll/IL-1 receptor domain containing adaptor protein (TIRAP), Toll/IL-1 receptor domain containing adaptor inducing interferon-beta (TRIF), and TRIF-related adaptor molecule (TRAM) [15-17] (Table 1). Triggering TLRs leads to activation of nuclear factor kappa B (NF $\kappa$ B) resulting in an upregulation of proinflammatory cytokines such as Tumour Necrosis Factor (TNF)- $\alpha$, IL-1 and IL-6, 
TABLE 1: Known TLRs, ligands for these TLRs and the relevant signalling pathways.

\begin{tabular}{lll}
\hline TLR & Recognises & Signals through \\
\hline 1 & Triacyl lipopeptides & TIRAP-MyD88 \\
2 & Tri- and diacyl lipopeptides & Mal/TIRAP-MyD88 \\
3 & dsRNA & TRIF-MyD88 independent \\
4 & LPS & Mal/TIRAP-MyD88 \& TRIF- MyD88 independent \\
5 & Flagellin & MyD88 \\
6 & Diacyl lipopeptides & TIRAP-MyD88 \\
7 & ssRNA & MyD88 \\
8 & ssRNA & MyD88 \\
9 & CpG-DNA (bacterial origin and host derived apoptotic DNA fragments) & MyD88 \\
10 & Undefined & Undefined \\
\hline
\end{tabular}

and/or activation of Interferon Response Factors (IRFs) which mediate transcription of Interferon (IFN) $\alpha$ and $\beta$ and subsequent downstream IFN-stimulated genes (ISGs). The specificity of the TLR signalling response is critically regulated by the various TIR-domain containing adapter molecules that associate with the TLR directly or colocalise with MyD88 [15-17].

MessengerRNA (mRNA) for all ten TLRs are expressed in murine livers, and expression levels can be modulated with corticosteroid treatment [18]. All ten human TLRs (TLR 1-10) are expressed in the liver with differential expression depending on the cell type [17]. While the liver is constantly exposed to bacterial microflora resident in the gastrointestinal (GI) tract via the portal circulation the inflammatory response is generally averted in the healthy liver by modulation of the innate immune response, referred to as "immune tolerance" (as reviewed by [19-21]). However, under conditions of chronic bacterial exposure, TLR expression may be similarly altered [22].

Primary cultured hepatocytes express TLRs 1-10 although the expression levels of TLRs $2-5$ are very low and may indicate immune tolerance or modulation of TLR expression in response to bacterial exposure [21].

Kupffer cells express TLRs 2, 3, 4, and 9 and are the first cell type in the liver to recognise translocated bacterial products, such as lipopolysaccharide (LPS) from the GI tract [21]. The physiological response of Kupffer cells to LPS in a normal healthy person is also hyporesponsive or "tolerant" [21]. Stimulation of TLR4 triggers a proinflammatory, profibrotic response with production of chemokines CCL2, 3 and 4 , adhesion molecules such as vascular cell adhesion molecule 1(V-CAM 1) and intercellular adhesion molecule 1 (I-CAM 1), transforming growth factor- $\beta$ (TGF $\beta)$ and upregulation of TLR2 expression [23-25].

Hepatic stellate cells (HSC) also express TLRs 4 and 9 $[23,24]$. The response of HSC to TLR9 ligation is profibrotic with enhanced collagen expression, but ligation may also limit fibrosis by inhibiting HSC migration [24].

Other main resident cell types in the liver also express TLRs including TLR2 -5 on human biliary epithelial cells [26] and TLR4 on rat liver sinusoidal endothelial cells (LSEC) [27]. Biliary epithelial cells and LSEC are generally tolerant to LPS stimulation of TLR4 [26, 27]. Liver dendritic cells (DC) and natural killer cells (NK) express a wide range of TLRs including TLR2, 3, 4, 7, and 9 on DCs and TLR1, 2, 3, $4,6,7$, and 9 on NK cells [17]. Unlike the resident monocytic kupffer cells, DCs and NKs respond appropriately to TLR4 ligation under normal physiological conditions [17].

\section{TLR Signalling, the Liver and HIV}

\subsection{Direct Effects}

3.1.1. HIV Infection of Liver Cells. Multiple cells in the liver can be infected with HIV. HIV RNA has been detected in primary human hepatocytes both ex vivo [28-30] and in vitro $[31,32]$, and we have recently shown that a number of hepatocyte cell lines are permissive to low level HIV infection in vitro [33]. Kupffer cells can be infected by HIV in vivo [28$30]$ and in vitro studies suggest that HIV infection of primary Kupffer cells leads to productive infection [31,32]. Binding of HIV or glycoprotein (gp)120 to CXCR4 expressed on HSCs led to an increase in CCL2 and other markers of HSC activation including alpha smooth muscle actin $(\alpha \mathrm{SMA})$ and TGF- $\beta[34,35]$.

3.1.2. Ligation of TLR7 and 8 by HIV. Given HIV RNA is also a TLR7/8 ligand [36] and hepatocytes express either TLR7, TLR8, or both, HIV may also directly activate TLRs in the liver, although to date this has not been explored.

The effects of HIV on TLR activation have been assessed in mononuclear cells from blood. Ligation of TLR8 by HIV RNA led to an increase in HIV replication and TNF- $\alpha$ production in monocytes from blood via activation of the MyD88-dependent NF $\kappa$ B pathway [37, 38]. Following HIV infection of monocytes or stimulation with single stranded (ss)HIV RNA the response to LPS stimulation was significantly enhanced with an increased production of proinflammatory cytokines including TNF- $\alpha$, consistent with a loss of "tolerance" [39]. Given Kupffer cells are of monocytic origin, they may also respond to HIV through activation of TLR7/8 (Figure 1). Therefore, if there was any low level persistence of HIV RNA in the liver, even in the setting of suppressive cART, this may potentially enhance the intrahepatic inflammatory responses to LPS. 


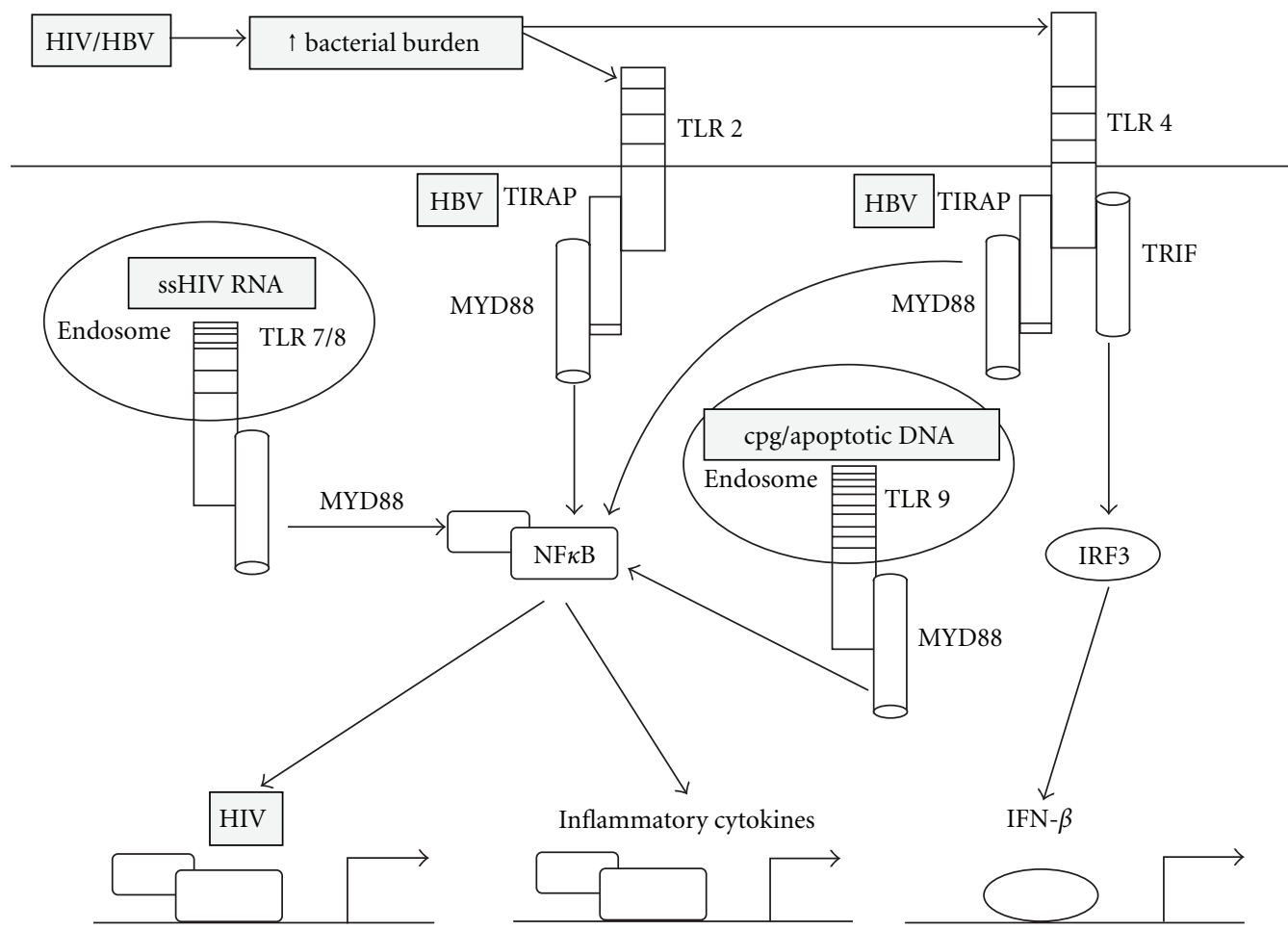

FIGURE 1: TLR signalling in the liver in the setting of HIV-HBV coinfection. HIV infection results in a significant increase in circulating LPS, potentially triggering an inflammatory response via activation of TLR4. ssHIV RNA can also activate TLR7 or 8 leading to an increase in NF- $\kappa$ B which can drive both HIV replication and production of proinflammatory cytokines and chemokines. HBV binds to and inhibits signalling through Mal/TIRAP which would inhibit proinflammatory responses in the coinfected liver. Increased LPS and/or increased hepatic apoptosis in the coinfected liver could also activate TLR9 resulting in further inflammation and HIV replication.

3.1.3. TLR9 and HIV. Other TLRs, such as TLR9, may also contribute to effects of HIV on the liver. Several studies have now shown TLR9 polymorphisms are associated with rapid disease progression [40, 41] and higher peak viral load [42] suggesting TLR9 ligation events are important drivers of inflammation during HIV infection.

TLR9 binds CpG-DNA of bacterial origin but more recently, it has been shown that TLR9 can also be activated by hepatocyte-derived apoptotic DNA fragments [24]. HIV can induce hepatocyte apoptosis in vitro via binding of gp120 to CXCR4 even in the absence of productive infection [43]. We have recently shown that intrahepatic apoptosis is increased in the setting of HIV-HBV coinfection using immunohistochemistry of liver biopsies [44]. Signalling through TLR9 has also been shown to suppress HIV replication in ex vivo lymphoid tissue blocks which correlated with production of chemokines such as CXCL-10 and 12 and CCL 3, 4, and 5 [45]. Therefore, indirect effects of HIV on TLR9 may also contribute to liver disease in the setting of HIV infection.

3.1.4. HIV and Dendritic Cells. Dendritic cells provide an important bridge between the innate and adaptive immune systems. Plasmacytoid DC ( $\mathrm{pDC}$ ) in particular secrete type I IFNs and proinflammatory cytokines following stimulation with either TLR7 or TLR9 that in turn activate T cells. During HIV infection, DC numbers and function are altered [46]. HIV gp120 has been shown to inhibit the pDC proinflammatory response to TLR9 ligation in vitro [47], and it has been demonstrated that ssHIV RNA activates pDCs via TLR7/8 ligation suggesting a mechanism whereby HIV drives chronic immune activation [36]. However, the inflammatory response of DCs from HIV-infected patients to TLR ligation is unclear and has been described as either reduced $[48,49]$ or unchanged [50,51]. It is not currently known how HIV or TLR ligation affects DCs of the liver.

3.2. Indirect Effects: HIV and LPS. HIV infection leads to a significant depletion of CD4 T cells in the GI tract which is associated with a significant increase in plasma LPS [52-54]. It is hypothesised that the increased LPS burden in the setting of HIV infection leads to activation of the innate immune response including monocyte/macrophages and DCs leading to a significant increase in IFN- $\alpha$, IL-6, and TNF- $\alpha$ [55]. There is also a significant association between LPS and circulating activated CD4 and CD8 T cells (as measured by expression of CD38 and HLA-DR) [53]. Exposure of peripheral blood mononuclear cells (PBMCs) to agonists of TLRs 3, 4,5 , and 9 in vitro resulted in activation of both memory and effecter CD4 and CD8 T cells, consistent with LPS also driving T-cell activation [56].

While it is currently unclear if elevated LPS plays a role in liver disease progression in HIV monoinfection, it is clear that elevated LPS is associated with, and in some settings contributes to, liver disease progression, for example, 
alcoholic liver disease [57], nonalcoholic fatty liver disease, nonalcoholic steatohepatitis [58-60], chronic hepatitis C virus (HCV) infection [61, 62], chronic HBV [61], and HIVHCV coinfection [63]. Increased intestinal permeability in HIV-HCV coinfected patients with cirrhosis was associated with an increase in circulating LPS and monocyte activation in blood and an increase in gene expression of hepatic TLR2 and TLR4 [22]. In addition, TNF- $\alpha$ expression was significantly correlated with hepatic inflammation as measured by histology [64].

$\mathrm{NF} \kappa \mathrm{B}$ is a common downstream component of the TLR signalling pathway. The HIV promoter, referred to as the long terminal repeat (LTR), contains two $\mathrm{NF} \kappa \mathrm{B}$ binding sites, and HIV transcription is significantly increased following $\mathrm{NF} \kappa \mathrm{B}$ binding [65]. This can be negatively regulated by nuclear receptors peroxisome proliferator-activated receptor gamma $(\operatorname{PPAR} \gamma)$ and liver X receptor (LXR) [66]. Several studies have shown that TLR activation by microbial products such as LPS or flagellin enhances HIV replication via an increase in $\mathrm{NF} \kappa \mathrm{B}[45,67]$, therefore, a positive feedback mechanism may exist whereby elevated circulating LPS drives HIV replication which may drive further $\mathrm{CD} 4 \mathrm{~T}$-cell depletion in turn driving further increases in LPS (Figure 1).

Strategies that target immune activation in HIV infection might also be potentially beneficial in the management of liver disease the setting of HIV infection. Amongst many antiactivation approaches that are currently being evaluated, hydroxychloroquine, a drug used to treat inflammatory arthritis, shows the most promise. Hydroxychloroquine reduces endosomal TLR signalling and has recently been evaluated in HIV-infected patients with reduced CD4 T-cell recovery [68]. In this study, hydroxychloroquine led to a significant decrease in plasma LPS and multiple other markers of immune activation and also led to enhanced CD4 T-cell recovery [68]. Further work is still required to better understand whether reducing immune activation in HIV-infected patients on cART will have an effect on liver disease outcomes.

\section{TLR Signalling in HIV-HBV Coinfection: Potential Interactions}

On average, $10 \%$ of patients infected with HIV are coinfected with hepatitis B virus. With the introduction of cART, which also includes agents active against both HIV and HBV (HBVactive HAART), liver-related mortality rates have reduced [1] but total and liver-related mortality still remain significantly elevated in HIV-HBV coinfected patients [6, 69].

In a mouse model of HBV infection, activation of TLRs 3 , 4, 5, 7, or 9 suppressed HBV replication [70] and in another study activation of TLR3 and TLR4 suppressed HBV replication via the MyD88-independent pathway [71]. Like most successful persistent viruses, HBV has evolved to adapt and even subvert the host innate immune response.

4.1. HBV and TLR2 and TLR4. Previous work by ourselves and others has shown that in chronic HBV monoinfection, TLR2 expression was downregulated on primary circulating monocytes in chronic HBV infection compared to uninfected controls [72-74]. Compared to HBeAg-negative patients, HBeAg positive patients had lower expression of TLR2 on monocytes, hepatocytes and kupffer cells [74]. This was associated with a decrease in TNF- $\alpha$ expression when either TLR4 or TLR2 was activated in vitro [74]. Most recently, in an hepatocyte cell line, we have shown that HBeAg binds to TIR-domain containing adapter-inducing interferon (TRIF)-related adapter molecule (TRAM) and MyD88-adapter like/Toll/IL-1 receptor domain containing adapter protein (Mal/TIRAP) to effectively inhibit signalling through TLR2 and TLR4 which may represent another strategy for HBV to evade the innate immune response [75].

In HIV-HBV coinfection, it is plausible that chronic exposure to elevated LPS could contribute to a loss of tolerance in TLR4 signalling in the liver. In combination with an impaired TLR2 signalling response mediated by HBV, the TRIF-dependent IFN response might be further enhanced (Figure 1). These interactions warrant further investigation using in vitro model systems.

4.2. HBV and TLR9. HBV has also been shown to impair TLR9 signalling in plasmacytoid DCs (pDCs) leading to reduced IFN- $\alpha$ production [76] which may be another mechanism for evasion of the immune system. TLR9 expression is increased in patients with chronic HBV compared to uninfected controls, and TLR9 expression significantly correlates with HBV DNA levels in plasma [77], suggesting a link between TLR9 expression and viral replication. Given the direct and indirect effects of HIV on TLR9, it is possible that, in the setting of HIV-HBV coinfection, TLR9 signalling in the liver by both HIV and HBV could result in an increase in chemokine production and a proinflammatory response.

\section{TLR Signalling in HIV-HCV Coinfection: Potential Interactions}

The role of TLR signalling in HCV infection has been reviewed elsewhere [23]. In brief, HCV has adapted multiple methods of evading the host innate immune response by downregulating signalling through TLRs 2, 4, 7, and 9 by binding to and interfering with MyD88 and TRIF in multiple cell types including hepatocytes, macrophages, and plasmacytoid (p)DCs [23]. HCV has also been shown to cleave mitochondrial antiviral signalling protein (MAVS) which forms part of the viral sensing TLR signalling cascade, inhibiting IFN- $\alpha / \beta$ production, and transcription of antiviral ISGs [78]. Recent studies have also shown a potential role for TLR7 and 9 agonists in promoting clearance of HCV [7981]. This would suggest that HCV would contribute to a reduction in the antiviral response in patients with HIVHCV coinfection.

We recently examined liver RNA and peripheral blood monocytes from patients with HCV monoinfection $(n=$ $46)$, HIV-HCV coinfection $(n=27)$, and HIV monoinfection $(n=20)$. We found that increasing Metavir inflammatory activity score was associated with increased hepatic TLR2 and TLR4 mRNA as measured by reverse transcriptase quantitative PCR (RT-qPCR) [64]. We also found a significant correlation between hepatic mRNA expression of 
TNF- $\alpha$ and TLR2 and TLR4. Interestingly, we found no differences in TLR mRNA or protein expression between HCV monoinfected, HIV-HCV coinfected, or HIV monoinfected patients [64].

Monocytes from HCV and HIV-HCV coinfected patients lack LPS tolerance and this lack of tolerance correlated with liver inflammation, as measured by elevated intrahepatic Kupffer cells activation markers CD163 and CD33 and elevated circulating aspartate aminotransferase [61-63]. One study suggested an impaired or tolerant response to TLR3 and TLR4 in monocytes from HIV-HCV-infected individuals when stimulated ex vivo, however, in this study high spontaneous secretion of IL- 6 and TNF- $\alpha$ were observed, suggesting a loss of tolerance in vivo [82]. Plasma levels of LPS in HIV-HCV-coinfected patients also correlated with severity of cirrhosis [63], where patients with LPS in the upper quartile $(>42 \mathrm{pg} / \mathrm{mL}$ ) had a 19 -fold higher risk of disease progression compared to patients with LPS in the lower quartile.

\section{Conclusion}

HIV infection of the liver could potentially enhance TLR signaling via a direct effect of either the virus itself or HIVrelated proteins as well as via an indirect effect on increased circulating LPS levels. Our understanding of how HIV affects TLR function is largely derived from studies in blood. Further studies are needed to define the effects of HIV on expression of TLR and functional TLR signaling in hepatic cells, specifically in the presence of HBV and HCV coinfection. These studies will be critical for the development of novel strategies to manage HIV-HBV and HIV-HCV coinfection that extend beyond the use of antivirals alone.

\section{Author's Contribution}

M. Crane, K. Visvanathan, and S. R. Lewin all contributed equally to writing this paper.

\section{Acknowledgments}

M. Crane is supported by an American Foundation for AIDS Research (AmFAR) Mathilde Krim Biomedical Fellowship; S. R. Lewin is supported by an NHMRC Practitioner Fellowship.

\section{References}

[1] C. Smith, "Factors associated with specific causes of death amongst HIV-positive individuals in the D:A:D study," AIDS, vol. 24, no. 10, pp. 1537-1548, 2010.

[2] R. Weber, "Liver-related deaths in persons infected with the human immunodeficiency virus the D:A:D study," Archives of Internal Medicine, vol. 166, no. 15, pp. 1632-1641, 2006.

[3] N. Bodsworth, B. Donovan, and B. N. Nightingale, "The effect of concurrent human immunodeficiency virus infection on chronic hepatitis B: a study of 150 homosexual men," Journal of Infectious Diseases, vol. 160, no. 4, pp. 577-582, 1989.

[4] J. F. Colin, D. Cazals-Hatem, M. A. Loriot et al., "Influence of human immunodeficiency virus infection on chronic hepatitis
B in homosexual men," Hepatology, vol. 29, no. 4, pp. 13061310, 1999.

[5] V. Soriano, E. Vispo, P. Labarga, J. Medrano, and P. Barreiro, "Viral hepatitis and HIV co-infection," Antiviral Research, vol. 85, no. 1, pp. 303-315, 2010.

[6] C. L. Thio, E. C. Seaberg, R. Skolasky Jr. et al., "HIV-1, hepatitis B virus, and risk of liver-related mortality in the Multicenter Cohort Study (MACS)," The Lancet, vol. 360, no. 9349, pp. 1921-1926, 2002.

[7] P. E. Chang, R. Miquel, J. L. Blanco et al., "Idiopathic portal hypertension in patients with HIV infection treated with highly active antiretroviral therapy," The American Journal of Gastroenterology, vol. 104, no. 7, pp. 1707-1714, 2009.

[8] N. Crum-Cianflone, A. Dilay, G. Collins et al., "Nonalcoholic fatty liver disease among HIV-infected persons," Journal of Acquired Immune Deficiency Syndromes, vol. 50, no. 5, pp. 464473, 2009.

[9] P. Ingiliz, M. A. Valantin, C. Duvivier et al., "Liver damage underlying unexplained transaminase elevation in human immunodeficiency virus-1 mono-infected patients on antiretroviral therapy," Hepatology, vol. 49, no. 2, pp. 436-442, 2009.

[10] I. Maida, P. Garcia-Gasco, G. Sotgiu et al., "Antiretroviralassociated portal hypertension: a new clinical condition? Prevalence, predictors and outcome," Antiviral Therapy, vol. 13, no. 1, pp. 103-107, 2008.

[11] G. Panos, L. Farouk, J. Stebbing et al., "Cryptogenic pseudocirrhosis: a new clinical syndrome of noncirrhotic portal hypertension (unassociated with advanced fibrosis) that can be detected by transient elastography in patients with HIV," Journal of Acquired Immune Deficiency Syndromes, vol. 52, no. 4, pp. 525-527, 2009.

[12] N. Crum-Cianflone, G. Collins, S. Medina et al., "Prevalence and factors associated with liver test abnormalities among human immunodeficiency virus-infected persons," Clinical Gastroenterology and Hepatology, vol. 8, no. 2, pp. 183-191, 2010.

[13] M. DallaPiazza, V. K. Amorosa, R. Localio, J. R. Kostman, and V. Lo Re III, "Prevalence and risk factors for significant liver fibrosis among HIV-monoinfected patients," BMC Infectious Diseases, vol. 10, article 116, 2010.

[14] H. Kovari, B. Ledergerber, M. Battegay et al., "Incidence and risk factors for chronic elevation of alanine aminotransferase levels in HIV-infected persons without hepatitis B or C virus co-infection," Clinical Infectious Diseases, vol. 50, no. 4, pp. 502-511, 2010.

[15] S. Akira, S. Uematsu, and O. Takeuchi, "Pathogen recognition and innate immunity," Cell, vol. 124, no. 4, pp. 783-801, 2006.

[16] L. A. J. O’Neill and A. G. Bowie, "The family of five: TIRdomain-containing adaptors in Toll-like receptor signalling," Nature Reviews Immunology, vol. 7, no. 5, pp. 353-364, 2007.

[17] E. Seki and D. A. Brenner, "Toll-like receptors and adaptor molecules in liver disease: update," Hepatology, vol. 48, no. 1, pp. 322-335, 2008.

[18] R. Broering, M. Montag, M. Jiang et al., "Corticosteroids shift the Toll-like receptor response pattern of primary-isolated murine liver cells from an inflammatory to an anti-inflammatory state," International Immunology, vol. 23, no. 9, pp. 537-544, 2011.

[19] I. N. Crispe, "Hepatic T cells and liver tolerance," Nature Reviews Immunology, vol. 3, no. 1, pp. 51-62, 2003.

[20] B. Gao, W. I. Jeong, and Z. Tian, "Liver: an organ with predominant innate immunity," Hepatology, vol. 47, no. 2, pp. 729-736, 2008. 
[21] R. F. Schwabe, E. Seki, and D. A. Brenner, "Toll-like receptor signaling in the liver," Gastroenterology, vol. 130, no. 6, pp. 1886-1900, 2006.

[22] M. Montes-de-Oca, M. J. Blanco, M. Marquez et al., "Haemodynamic derangement in human immunodeficiency virusinfected patients with hepatitis $\mathrm{C}$ virus-related cirrhosis: the role of bacterial translocation," Liver International, vol. 31, no. 6, pp. 852-860, 2011.

[23] E. Seki, S. De Minicis, C. H. Österreicher et al., "TLR4 enhances TGF- $\beta$ signaling and hepatic fibrosis," Nature Medicine, vol. 13, no. 11, pp. 1324-1332, 2007.

[24] A. Watanabe, A. Hashmi, D. A. Gomes et al., "Apoptotic hepatocyte DNA inhibits hepatic stellate cell chemotaxis via toll-like receptor 9," Hepatology, vol. 46, no. 5, pp. 1509-1518, 2007.

[25] Y. H. Paik, R. F. Schwabe, R. Bataller, M. P. Russo, C. Jobin, and D. A. Brenner, "Toll-like receptor 4 mediates inflammatory signaling by bacterial lipopolysaccharide in human hepatic stellate cells," Hepatology, vol. 37, no. 5, pp. 1043-1055, 2003.

[26] K. Harada, K. Isse, and Y. Nakanuma, "Interferon $\gamma$ accelerates NF- $\kappa$ B activation of biliary epithelial cells induced by Toll-like receptor and ligand interaction," Journal of Clinical Pathology, vol. 59, no. 2, pp. 184-190, 2006.

[27] A. Uhrig, R. Banafsche, M. Kremer et al., "Development and functional consequences of LPS tolerance in sinusoidal endothelial cells of the liver," Journal of Leukocyte Biology, vol. 77, no. 5, pp. 626-633, 2005.

[28] Y. Z. Cao, D. Dieterich, P. A. Thomas, Y. X. Huang, M. Mirabile, and D. D. Ho, "Identification and quantitation of HIV-1 in the liver of patients with AIDS," AIDS, vol. 6, no. 1, pp. 65-70, 1992.

[29] C. Housset, O. Boucher, P. M. Girard et al., "Immunohistochemical evidence for human immunodeficiency virus-1 infection of liver Kupffer cells," Human Pathology, vol. 21, no. 4, pp. 404-408, 1990.

[30] F. T. Hufert, J. Schmitz, M. Schreiber, H. Schmitz, P. Racz, and D. D. Laer, "Human Kupffer cells infected with HIV-1 in vivo," Journal of Acquired Immune Deficiency Syndromes, vol. 6, no. 7, pp. 772-777, 1993.

[31] J. L. Gendrault, A. M. Steffan, M. P. Schmitt, D. Jaeck, A. M. Aubertin, and A. Kirn, "Interaction of cultured human Kupffer cells with HIV-infected CEM cells: an electron microscopic study," Pathobiology, vol. 59, no. 4, pp. 223-226, 1991.

[32] M. P. Schmitt, J. L. Gendrault, C. Schweitzer et al., "Permissivity of primary cultures of human Kupffer cells for HIV-1," AIDS Research and Human Retroviruses, vol. 6, no. 8, pp. 987991, 1990.

[33] D. M. Iser, N. Warner, P. A. Revill et al., "Coinfection of hepatic cell lines with human immunodeficiency virus and hepatitis $B$ virus leads to an increase in intracellular hepatitis B surface antigen," Journal of Virology, vol. 84, no. 12, pp. 5860-5867, 2010.

[34] V. C. Asensio, J. Maier, R. Milner et al., "Interferon-independent, human immunodeficiency virus type 1 gp120-mediated induction of CXCL10/IP-10 gene expression by astrocytes in vivo and in vitro," Journal of Virology, vol. 75, no. 15, pp. 70677077, 2001.

[35] A. C. Tuyama, F. Hong, Y. Saiman et al., "Human immunodeficiency virus (HIV)-1 infects human hepatic stellate cells and promotes collagen I and monocyte chemoattractant protein-1 expression: implications for the pathogenesis of HIV/hepatitis C virus-induced liver fibrosis," Hepatology, vol. 52, no. 2, pp. 612-622, 2010.
[36] A. Meier, G. Alter, N. Frahm et al., "MyD88-dependent immune activation mediated by human immunodeficiency virus type 1-encoded Toll-like receptor ligands," Journal of Virology, vol. 81, no. 15, pp. 8180-8191, 2007.

[37] E. Schlaepfer, A. Audigé, H. Joller, and R. F. Speck, “TLR7/8 triggering exerts opposing effects in acute versus latent HIV infection," Journal of Immunology, vol. 176, no. 5, pp. 28882895, 2006.

[38] E. Schlaepfer and R. F. Speck, "TLR8 activates HIV from latently infected cells of myeloid-monocytic origin directly via the MAPK pathway and from latently infected $\mathrm{CD} 4^{+} \mathrm{T}$ cells indirectly via TNF- $\alpha$, Journal of Immunology, vol. 186, no. 7, pp. 4314-4324, 2011.

[39] M. W. Mureith, J. J. Chang, J. D. Lifson, T. Ndung'U, and M. Altfeld, "Exposure to HIV-1-encoded Toll-like receptor 8 ligands enhances monocyte response to microbial encoded Toll-like receptor $2 / 4$ ligands," AIDS, vol. 24, no. 12, pp. 18411848, 2010.

[40] P. Y. Bochud, M. Hersberger, P. Taffé et al., "Polymorphisms in Toll-like receptor 9 influence the clinical course of HIV-1 infection," AIDS, vol. 21, no. 4, pp. 441-446, 2007.

[41] N. Soriano-Sarabia, A. Vallejo, R. Ramirez-Lorca et al., "Influence of the Toll-like receptor 9 1635A/G polymorphism on the CD4 count, HIV viral load, and clinical progression," Journal of Acquired Immune Deficiency Syndromes, vol. 49, no. 2, pp. 128-135, 2008.

[42] S. O. Pine, M. J. McElrath, and P. Y. Bochud, "Polymorphisms in toll-like receptor 4 and toll-like receptor 9 influence viral load in a seroincident cohort of HIV-1-infected individuals," AIDS, vol. 23, no. 18, pp. 2387-2395, 2009.

[43] S. R. Vlahakis, A. Villasis-Keever, T. S. Gomez, G. D. Bren, and C. V. Paya, "Human immunodeficiency virus-induced apoptosis of human hepatocytes via CXCR4," Journal of Infectious Diseases, vol. 188, no. 10, pp. 1455-1460, 2003.

[44] D. M. Iser, A. Avihingsanon, N. Wisedopas et al., "Increased intrahepatic apoptosis but reduced immune activation in HIV-HBV co-infected patients with advanced immunosuppression,” AIDS, vol. 25, no. 2, pp. 197-205, 2011.

[45] B. Brichacek, C. Vanpouille, Y. Kiselyeva et al., "Contrasting roles for TLR ligands in HIV-1 pathogenesis," PLoS One, vol. 5, no. 9, Article ID e12831, 2010.

[46] J. Chehimi, E. Papasavvas, C. Tomescu et al., "Inability of plasmacytoid dendritic cells to directly lyse HIV-infected autologous $\mathrm{CD}^{+} \mathrm{T}$ cells despite induction of tumor necrosis factor-related apoptosis-inducing ligand," Journal of Virology, vol. 84, no. 6, pp. 2762-2773, 2010.

[47] E. Martinelli, C. Cicala, D. Van Ryk et al., "HIV-1 gp120 inhibits TLR9-mediated activation and IFN- $\alpha$ secretion in plasmacytoid dendritic cells," Proceedings of the National Academy of Sciences of the United States of America, vol. 104, no. 9, pp. 3396-3401, 2007.

[48] N. L. Yonkers, B. Rodriguez, R. Asaad, M. M. Lederman, and D. D. Anthony, "Systemic immune activation in HIV infection is associated with decreased MDC responsiveness to TLR ligand and inability to activate naive CD4 T-cells," PLoS One, vol. 6, no. 9, Article ID e23884, 2011.

[49] S. Nowroozalizadeh, F. Månsson, Z. D. Silva et al., "Studies on toll-like receptor stimuli responsiveness in HIV-1 and HIV-2 infections," Cytokine, vol. 46, no. 3, pp. 325-331, 2009.

[50] R. T. Lester, X. D. Yao, T. B. Ball et al., "Toll-like receptor expression and responsiveness are increased in viraemic HIV1 infection," AIDS, vol. 22, no. 6, pp. 685-694, 2008. 
[51] R. Sabado, M. O’Brien, A. Subedi et al., "Evidence of dysregulation of dendritic cells in primary HIV infection," Blood, vol. 116, no. 19, pp. 3839-3852, 2010.

[52] J. M. Brenchley, M. Paiardini, K. S. Knox et al., "Differential Th17 CD4 T-cell depletion in pathogenic and nonpathogenic lentiviral infections," Blood, vol. 112, no. 7, pp. 2826-2835, 2008.

[53] J. M. Brenchley, D. A. Price, T. W. Schacker et al., "Microbial translocation is a cause of systemic immune activation in chronic HIV infection," Nature Medicine, vol. 12, no. 12, pp. 13651371, 2006.

[54] A. Prendergast, J. G. Prado, Y. H. Kang et al., "HIV-1 infection is characterized by profound depletion of CD161+ Th17 cells and gradual decline in regulatory T cells," AIDS, vol. 24 , no. 4 , pp. 491-502, 2010.

[55] J. C. Tilton, A. J. Johnson, M. R. Luskin et al., "Diminished production of monocyte proinflammatoiy cytokines during human immunodeficiency virus viremia is mediated by type I interferons," Journal of Virology, vol. 80, no. 23, pp. 1148611497, 2006.

[56] N. Funderburg, A. A. Luciano, W. Jiang, B. Rodriguez, S. F. Sieg, and M. M. Lederman, "Toll-like receptor ligands induce human T cell activation and death, a model for HIV pathogenesis," PLoS One, vol. 3, no. 4, Article ID e1915, 2008.

[57] P. Mathurin, Q. G. Deng, A. Keshavarzian, S. Choudhary, E. W. Holmes, and H. Tsukamoto, "Exacerbation of alcoholic liver injury by enteral endotoxin in rats," Hepatology, vol. 32, no. 5, pp. 1008-1017, 2000.

[58] A. Abu-Shanab and E. M. Quigley, "The role of the gut microbiota in nonalcoholic fatty liver disease," Nature Reviews Gastroenterology \& Hepatology, vol. 7, no. 12, pp. 691-701, 2010.

[59] N. Lanthier, O. Molendi-Coste, Y. Horsmans, N. Van Rooijen, P. D. Cani, and I. A. Leclercq, "Kupffer cell activation is a causal factor for hepatic insulin resistance," American Journal of Physiology, vol. 298, no. 1, pp. G107-G116, 2010.

[60] Y. T. Zhan and W. An, "Roles of liver innate immune cells in nonalcoholic fatty liver disease," World Journal of Gastroenterology, vol. 16, no. 37, pp. 4652-4660, 2010.

[61] N. G. Sandler, C. Koh, A. Roque et al., "Host response to translocated microbial products predicts outcomes of patients with HBV or HCV infection," Gastroenterology, vol. 141, no. 4, pp. 1220-1230.e3, 2011.

[62] A. Dolganiuc, O. Norkina, K. Kodys et al., "Viral and host factors induce macrophage activation and loss of Toll like receptor tolerance in chronic HCV infection," Gastroenterology, vol. 133, no. 5, pp. 1627-1636, 2007.

[63] A. Balagopal, F. H. Philp, J. Astemborski et al., "Human immunodeficiency virus-related microbial translocation and progression of hepatitis C," Gastroenterology, vol. 135, no. 1, pp. 226-233, 2008.

[64] M. D. Berzsenyi, S. K. Roberts, S. Preiss et al., "Hepatic TLR2 \& TLR4 expression correlates with hepatic inflammation and TNF- $\alpha$ in HCV \& HCV/HIV infection," Journal of Viral Hepatitis, vol. 18, no. 12, pp. 852-860, 2011.

[65] S. A. Williams, H. Kwon, L. F. Chen, and W. C. Greene, "Sustained induction of NF- $\kappa \mathrm{B}$ is required for efficient expression of latent human immunodeficiency virus type 1," Journal of Virology, vol. 81, no. 11, pp. 6043-6056, 2007.

[66] T. M. Hanley and G. A. Viglianti, "Nuclear receptor signaling inhibits HIV-1 replication in macrophages through multiple trans-repression mechanisms," Journal of Virology, vol. 85, no. 20, pp. 10834-10850, 2011.
[67] V. H. Ferreira, A. Nazli, G. Khan et al., "Endometrial epithelial cell responses to coinfecting viral and bacterial pathogens in the genital tract can activate the HIV-1 LTR in an NF $\kappa$ B-and AP-1-dependent manner," Journal of Infectious Diseases, vol. 204, no. 2, pp. 299-308, 2011.

[68] S. Piconi, S. Parisotto, G. Rizzardini et al., "Hydroxychloroquine drastically reduces immune activation in HIV-infected, antiretroviral therapy-treated immunologic nonresponders," Blood, vol. 118, no. 12, pp. 3263-3272, 2011.

[69] C. J. Hoffmann, E. C. Seaberg, S. Young et al., "Hepatitis B and long-term HIV outcomes in coinfected HAART recipients," AIDS, vol. 23, no. 14, pp. 1881-1889, 2009.

[70] M. Isogawa, M. D. Robek, Y. Furuichi, and F. V. Chisari, “Tolllike receptor signaling inhibits hepatitis B virus replication in vivo," Journal of Virology, vol. 79, no. 11, pp. 7269-7272, 2005.

[71] J. Wu, M. Lu, Z. Meng et al., "Toll-like receptor-mediated control of HBV replication by nonparenchymal liver cells in mice," Hepatology, vol. 46, no. 6, pp. 1769-1778, 2007.

[72] Z. Chen, Y. Cheng, Y. Xu et al., "Expression profiles and function of Toll-like receptors 2 and 4 in peripheral blood mononuclear cells of chronic hepatitis B patients," Clinical Immunology, vol. 128, no. 3, pp. 400-408, 2008.

[73] S. M. Riordan, N. Skinner, J. Kurtovic, S. Locarnini, and K. Visvanathan, "Reduced expression of Toll-like receptor 2 on peripheral monocytes in patients with chronic hepatitis B," Clinical and Vaccine Immunology, vol. 13, no. 8, pp. 972-974, 2006.

[74] K. Visvanathan, N. A. Skinner, A. J. V. Thompson et al., "Regulation of Toll-like receptor-2 expression in chronic hepatitis B by the precore protein," Hepatology, vol. 45, no. 1, pp. 102-110, 2007.

[75] T. Lang, C. Lo, N. Skinner, S. Locarnini, K. Visvanathan, and A. Mansell, "The Hepatitis B e antigen (HBeAg) targets and suppresses activation of the Toll-like receptor signaling pathway," Journal of Hepatology, vol. 55, no. 4, pp. 762-769, 2011.

[76] Q. Xie, H. C. Shen, N. N. Jia et al., "Patients with chronic hepatitis B infection display deficiency of plasmacytoid dendritic cells with reduced expression of TLR9," Microbes and Infection, vol. 11, no. 4, pp. 515-523, 2009.

[77] N. Xu, H. P. Yao, Z. Sun, and Z. Chen, “Toll-like receptor 7 and 9 expression in peripheral blood mononuclear cells from patients with chronic hepatitis B and related hepatocellular carcinoma," Acta Pharmacologica Sinica, vol. 29, no. 2, pp. 239-244, 2008.

[78] P. Bellecave, M. Sarasin-Filipowicz, O. D. Sarasin-Filipowicz et al., "Cleavage of mitochondrial antiviral signaling protein in the liver of patients with chronic hepatitis $\mathrm{C}$ correlates with a reduced activation of the endogenous interferon system," Hepatology, vol. 51, no. 4, pp. 1127-1136, 2010.

[79] J. G. McHutchison, B. R. Bacon, S. C. Gordon et al., "Phase 1B, randomized, double-blind, dose-escalation trial of CPG 10101 in patients with chronic hepatitis C virus," Hepatology, vol. 46, no. 5, pp. 1341-1349, 2007.

[80] Y. Horsmans, T. Berg, J. P. Desager et al., "Isatoribine, an agonist of TLR7, reduces plasma virus concentration in chronic hepatitis C infection," Hepatology, vol. 42, no. 3, pp. 724-731, 2005.

[81] J. F. Bergmann, J. De Bruijne, D. M. Hotho et al., "Randomised clinical trial: anti-viral activity of ANA773, an oral inducer 
of endogenous interferons acting via TLR7, in chronic HCV," Alimentary Pharmacology \& Therapeutics, vol. 34, no. 4, pp. 443-453, 2011.

[82] M. C. Villacres, O. Literat, M. DeGiacomo, W. Du, T. Frederick, and A. Kovacs, "Defective response to Toll-like receptor 3 and 4 ligands by activated monocytes in chronic hepatitis $\mathrm{C}$ virus infection," Journal of Viral Hepatitis, vol. 15, no. 2, pp. 137-144, 2008. 


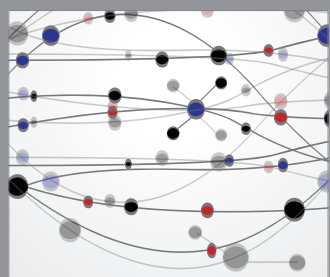

The Scientific World Journal
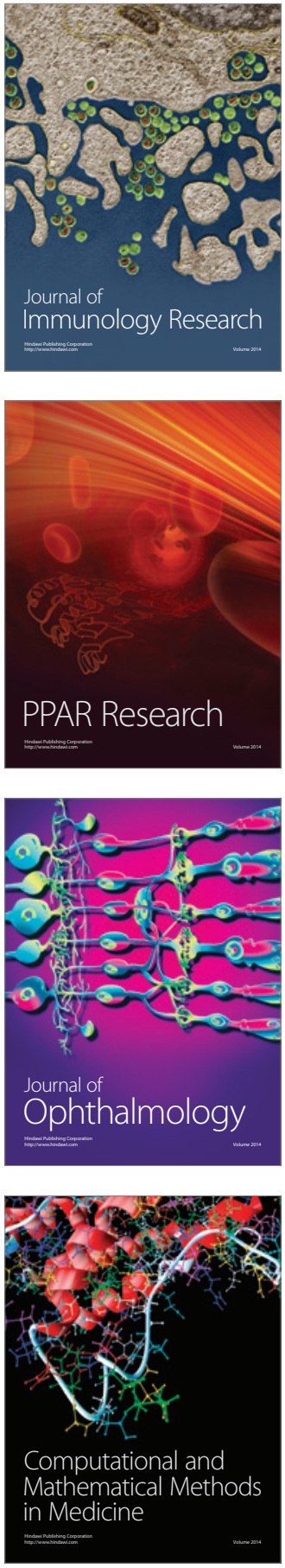

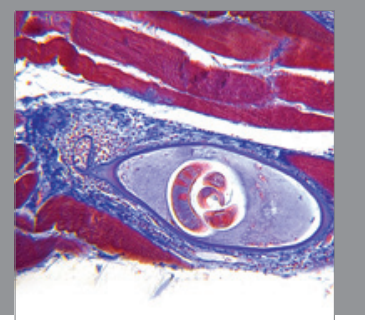

Gastroenterology

Research and Practice
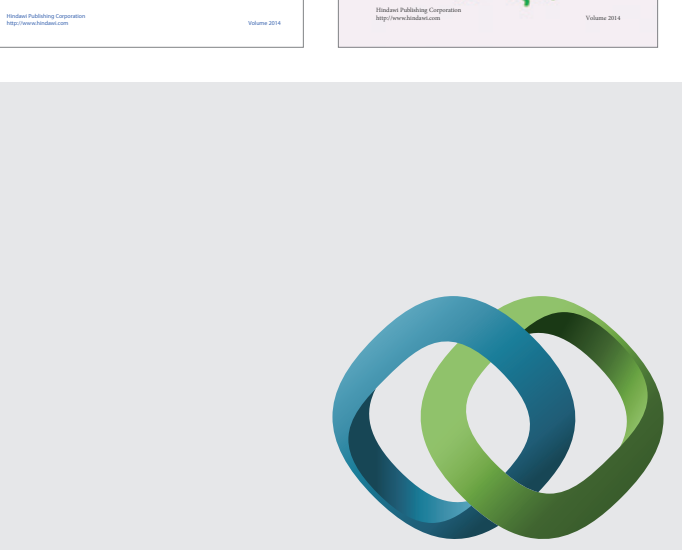

\section{Hindawi}

Submit your manuscripts at

http://www.hindawi.com
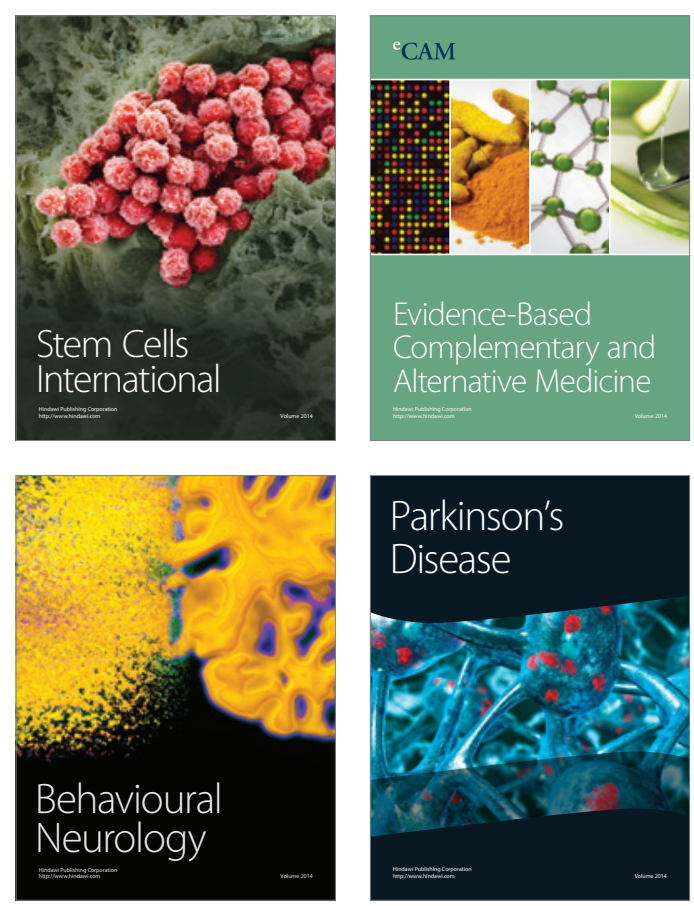

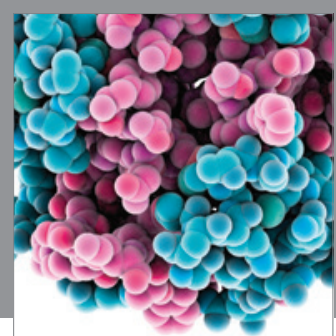

Journal of
Diabetes Research

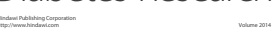

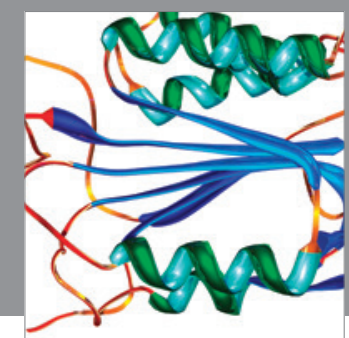

Disease Markers
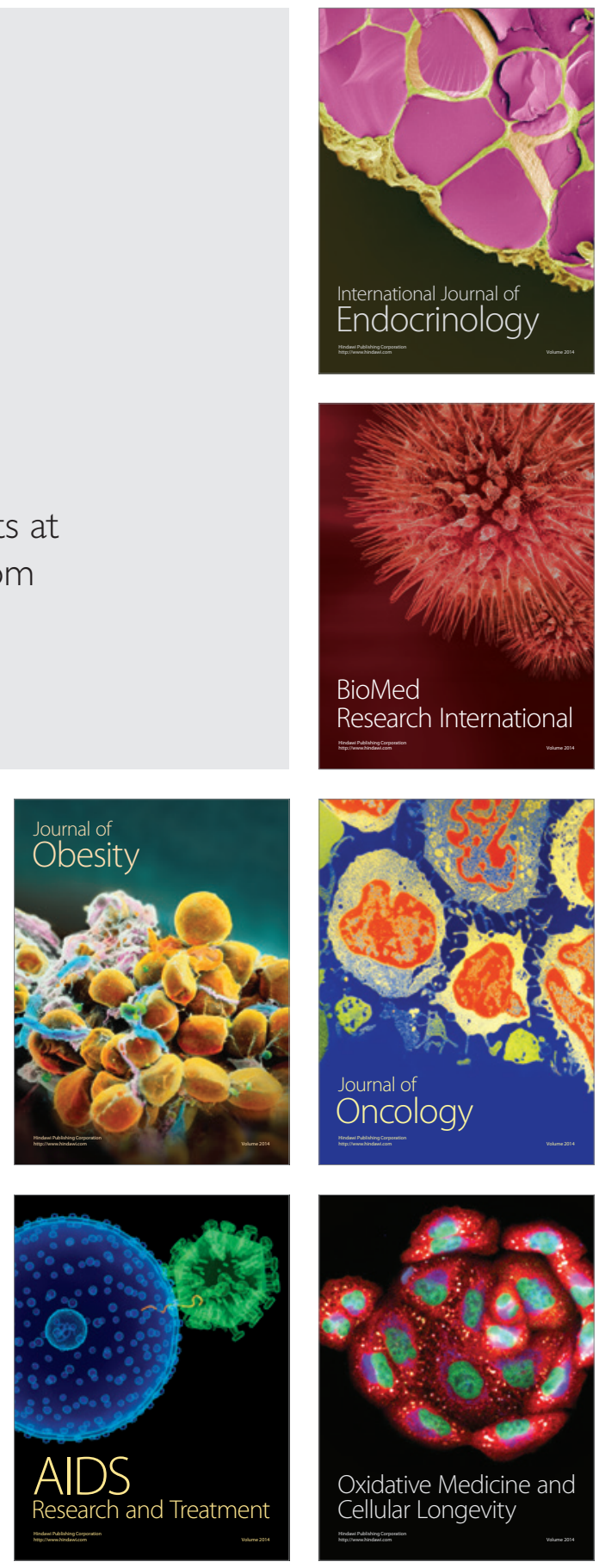